LA-UR-01-6139

Approved for public release; distribution is unlimited.

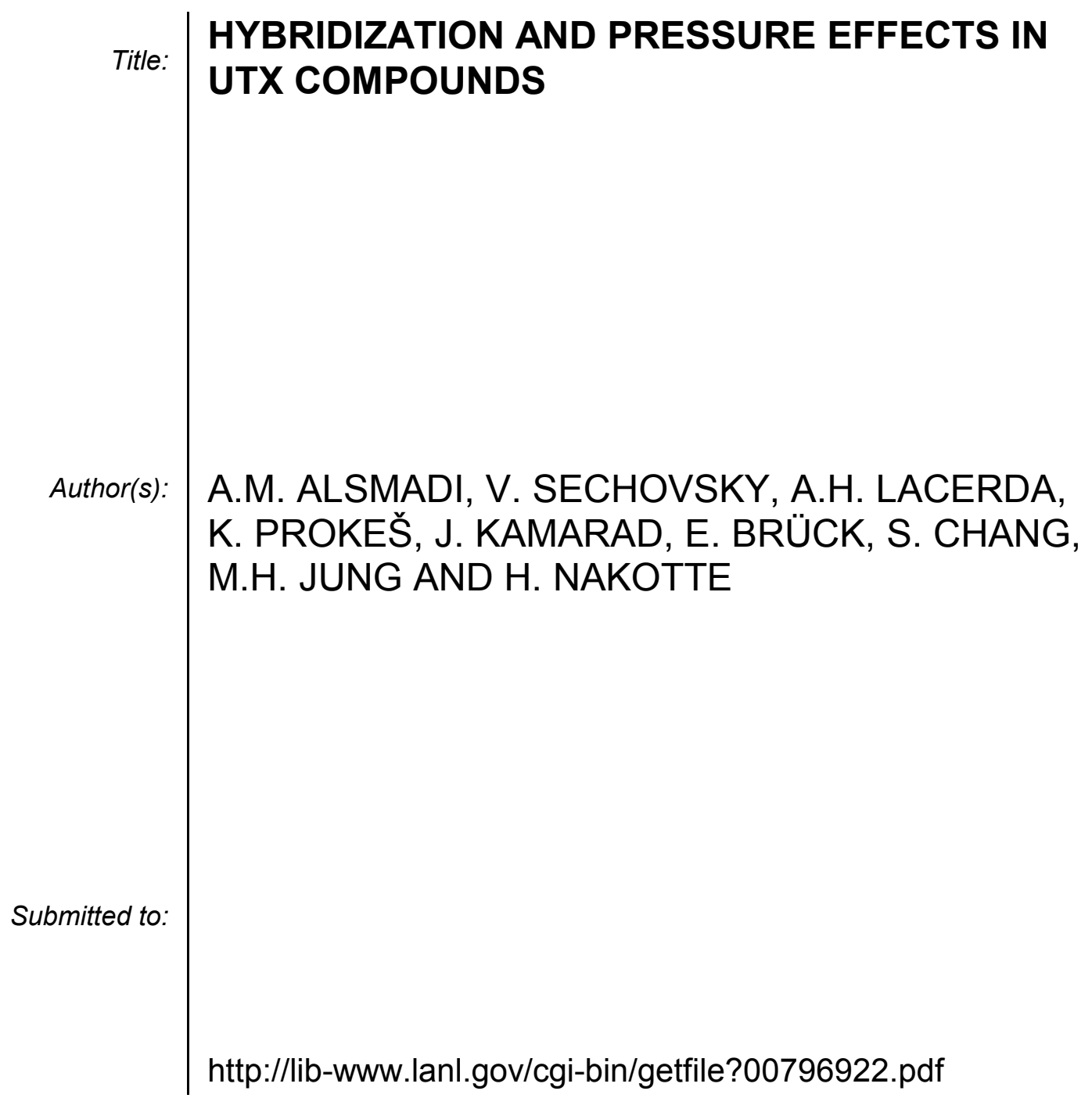

Los Alamos National Laboratory, an affirmative action/equal opportunity employer, is operated by the University of California for the U.S. Department of Energy under contract W-7405-ENG-36. By acceptance of this article, the publisher recognizes that the U.S. Government retains a nonexclusive, royaltyfree license to publish or reproduce the published form of this contribution, or to allow others to do so, for U.S. Government purposes. Los Alamos National Laboratory requests that the publisher identify this article as work performed under the auspices of the U.S. Department of Energy. Los Alamos National Laboratory strongly supports academic freedom and a researcher's right to publish; as an institution, however, the Laboratory does not endorse the viewpoint of a publication or guarantee its technical correctness. 


\title{
HYBRIDIZATION AND PRESSURE EFFECTS IN UTX COMPOUNDS
}

\author{
A.M. ALSMADI ${ }^{1,2}$, V. SECHOVSKY ${ }^{3}$, A.H. LACERDA ${ }^{4}$, K. PROKES $\breve{S}^{3,5}$, J. KAMARAD $^{6}$, E. \\ BRÜCK $^{7}$, S. CHANG ${ }^{1}$, M.H. JUNG ${ }^{1,4}$ AND H. NAKOTTE ${ }^{1,2}$ \\ ${ }^{1}$ Physics Department, New Mexico State University, Las Cruces, NM 88003 \\ ${ }^{2}$ Los Alamos Neutron Scattering Center, Los Alamos National Laboratory, Los Alamos, New Mexico \\ 87545 \\ ${ }^{3}$ Department of Electronic Structures, Charles University, 12116 Prague 2, The CzechRepublic \\ ${ }^{4}$ National High Magnetic Field Laboratory, Pulse Field Facility, Los Alamos National Laboratory, Los \\ Alamos, NM 87545 \\ ${ }^{5}$ Hahn-Meitner-Institute, SF-2, Glienickerstrasse 100, D-141 09 Berlin, Germany \\ ${ }^{6}$ Laboratory of High Pressure, Institute of Physics, AS CR, Czech Academy of Sciences, Cukrovarnicka 10, \\ 16253 Praha 6, Czech Republic \\ ${ }^{7}$ Van der Waals-Zeeman Instituut, Universiteit van Amsterdam, Valckenierstraat 65, 1018 XE Amsterdam, \\ The Netherlands
}

\begin{abstract}
:
The UTX intermetallic compound $(\mathrm{T}=$ transition metal and $\mathrm{X}=p$-electron element) were found to crystallize mainly in two large groups, the orthorhombic TiNiSi and the hexagonal $\mathrm{ZrNiAl}$ structure. For both groups, magnetic-ordering phenomena depend sensitively on the 5 f-ligand hybridization. The 5 f-ligand hybridization is very sensitive to the interatomic distances, which can be effectively controlled by external pressure. Here, we have summarized existing and new pressure studies on various single-crystalline UTX compounds ( $\mathrm{T}=\mathrm{Co}, \mathrm{Ni}, \mathrm{Ir} ; \mathrm{X}=\mathrm{Al}, \mathrm{Ga}, \mathrm{Ge})$. We performed magnetoresistance studies in magnetic fields up to $18 \mathrm{~T}$ under external hydrostatic pressure up to $10 \mathrm{kbar}$. The observed dependencies of the ordering temperatures and the critical fields are compared with the different contributions of the $5 f$-ligand hybridization, which were deduced from tight-binding calculations. We find relatively weak pressure dependence in compounds, where $5 f$ electrons are more localized, while substantially large pressure effects are found in the more itinerant systems.
\end{abstract}

KEYWORDS: pressure studies, tight-binding calculations, hybridization effects

\section{Corresponding author:}

Abdel K. Alsmadi

Physics Department MSC 3D

New Mexico State University

Las Cruces NM 88003

Phone: 1-505-646-5039

Fax: 1-505-646-1934

e-mail:aalsmadi@nmsu.edu 
Uranium compounds have attracted a great deal of attention in the past two decades because of the specific nature of $\mathrm{U} 5 f$-electron magnetism. The $f$ electrons of $\mathrm{U}$ are found to be intermediate between the delocalized $d$ electrons of the transition metal and the well-localized $4 f$ electrons of the lanthanide. Two mechanisms are known to lead to delocalization the $5 f$ electrons, the direct $5 f-5 f$ overlap and the $5 f$-ligand hybridization. ${ }^{1,2}$ Comprehensive studies have revealed a large variety of magnetic phenomena starting from weak paramagnetism to various types of (sometimes unusual) long-range magnetic ordering depending on the degree of the $5 f$-ligand hybridization. Large $5 f$-ligand hybridization leads to a delocalization of the $f$ electrons, which ultimately leads to a suppression of magnetic moments. On the other hand, in most cases some $5 f$ ligand hybridization is needed to promote inter-ionic exchange, which confirms a magnetic order ground state. The $5 f$ ligand hybridization depends on several parameters (geometrical surrounding of the $5 f$ atom, coordination, interatomic distances, etc.), and it is determined by the overlap of the respective wavefunctions. The interatomic distances and thus the overlap of wavefunctions can be effectively changed by external pressure.

UTX compounds $(\mathrm{T}=$ transition metal, $\mathrm{X}=p$-electron element) were found to crystallize mainly in two large groups of crystal structures, the orthorhombic TiNiSi structure and the hexagonal $\mathrm{ZrNiAl}$ structure. For both groups, extensive bulk studies revealed a huge magneto-crystalline anisotropy, which is of easy-axis type (c-axis) in the $\mathrm{ZrNiAl}$-structure and easy-plane type ( $b-c$ plane) for the TiNiSi-structure compounds. ${ }^{3-6}$ The huge magnetic anisotropy originates in strong spin-orbit interactions and the presence of large orbital moments, and the magnetic anisotropy was tentatively attributed to the hybridization-mediated anisotropic two-ion interaction.

In this contribution, we have summarized the effect of external pressure on singlecrystalline UTX compounds $(\mathrm{T}=\mathrm{Co}, \mathrm{Ni}, \mathrm{Ir} ; \mathrm{X}=\mathrm{Al}, \mathrm{Ga}, \mathrm{Ge})$ studied to date. The compounds with $\mathrm{X}=\mathrm{Al}$ or Ga crystallize in the hexagonal ZrNiAl structure, and the compounds with $\mathrm{X}=\mathrm{Ge}$ crystallize in the orthorhombic TiNiSi structure. UCoGa, UIrAl and UIrGa are ferromagnets at low temperatures, and UCoGe is a paramagnet.' Antiferromagnetic ground states were established in UNiAl, UNiGa, UNiGe and UIrGe, and UCoAl exhibits metamagnetic behavior at low temperatures. ${ }^{1}$ Our pressure studies have been limited to single-crystalline UTX compounds that exhibit antiferromagnetic or metamagnetic ground states.

Electrical-resistance and magnetoresistance studies were performed in order to gain information on the pressure dependence of the magnetic-ordering temperature and critical fields, respectively. Most of our experimental studies (UNiAl, UNiGe, UIrGe) were done in the 20-T superconducting magnet at the Pulse Field Facility, NHMFL, Los Alamos National Laboratory, under hydrostatic pressure up to $10 \mathrm{kbar}$ using a $\mathrm{Cu}-\mathrm{Be}$ pressure cell with mineral oil as the pressure-transmitting medium. The results on UIrGe are shown for the first time, while some of our results on UNiGe and UNiAl have been published previously. ${ }^{8-10}$ Here, we extracted the pressure gradients of the ordering temperatures $\mathrm{dT}_{\mathrm{N}} / \mathrm{dp}$ and the critical fields $\mathrm{dB}_{\mathrm{c}} / \mathrm{dp}$ as well as the critical pressure $\mathrm{p}_{\mathrm{c}}$ (determined by an extrapolation of pressure to $\mathrm{T}_{\mathrm{N}}=0 \mathrm{~K}$ ) for these three compounds and compared them with the published ones on $\mathrm{UNiGa}^{11}$ and UCoAl. ${ }^{12,13}$ Here, we are aware of the fact that the results on the values of $p_{c}$ can be valid only under the assumption that the compressibility remains constant. In most of $\mathrm{d}$ materials the compressibility is varying (usually getting lower with increasing pressure). Therefore, we propose the 
values of $\mathrm{p}_{\mathrm{c}}$ to be only a rough estimate for our materials. Direct measurement up to higher pressure is desired to resolve this issue.

Our experiments on UIrGe were performed on a single crystal extracted from the same batch as used in previous studies. ${ }^{14}$ We performed temperature scans at various pressures in fixed fields and field scans at various pressures with fixed temperatures. As an example, we display the temperature dependence of the magnetoresistance at various fixed fields under an applied pressure of $6 \mathrm{kbar}$ in Fig. 1. In Fig. 2, the low-temperature behavior of the electric resistance in UIrGe is shown for different values of pressure up to $9 \mathrm{kbar}$. We find that both $\mathrm{T}_{\mathrm{N}}$ and the transition fields (see inset of Fig. 2) decrease slightly with increasing pressure. As an aside, we find that pressure seems to promote a less metallic ground state, i.e. the increase of the low-temperature resistance is more pronounced compared to the ambient-pressure result. This finding could explain the apparent differences in the temperature dependence of the electrical resistance for polycrystalline $^{14}$ (metallic behavior below $\mathrm{T}_{\mathrm{N}}$ ) and single-crystalline UIrGe (increasing resistance below $\mathrm{T}_{\mathrm{N}}$. On the other hand, we find that there is no further enhancement of the low-temperature increase of the electrical resistance between 6 and $9 \mathrm{kbar}$. The curves are almost parallel with slightly lower resistance values in 9 kbar due to the suppression of $\mathrm{T}_{\mathrm{N}}$. For UNiSn, it has been established that the ground-state properties can be altered by the application of relatively small strain fields ${ }^{15}$. It is possible that internal strains have strong effects also in UIrGe. We speculate that internal pressure effects in singlecrystalline UIrGe are needed to promote an increase in the low-temperature resistance. In polycrystalline UIrGe, on the other hand, grain boundaries may hamper the propagation of such internal pressure effects, which in turn results a more metallic resistance behavior.

In table 1, we have summarized the ordering temperatures $T_{N}$, the pressure rates $\mathrm{dT}_{\mathrm{N}} / \mathrm{dp}, \mathrm{dB}_{\mathrm{c}} / \mathrm{dp}$ and critical pressure $\mathrm{p}_{\mathrm{c}}$ for various UTX compounds. Inspection of the values in table 1 , we find that $\mathrm{dT}_{\mathrm{N}} / \mathrm{dp}$ is larger in UIrGe and UNiAl compared to UNiGe and $\mathrm{UNiGa}$, which can be taken as evidence that the $5 f$ electrons are more delocalized in these two compounds. UCoAl has a metamagnetic ground state, thus there is no value for $\mathrm{dT}_{\mathrm{N}} / \mathrm{dp}$. From the values for $\mathrm{p}_{\mathrm{c}}$, however, we may conclude that UNiAl is more localized than UIrGe and UCoAl, but more delocalized than UNiGe and UNiGa. While $\mathrm{dT}_{\mathrm{N}} / \mathrm{dp}$ is negative for all compounds, surprisingly we find opposite signs in $\mathrm{dT}_{N} / d p$ and $\mathrm{dB}_{\mathrm{c}} / \mathrm{dp}$ in the two localized representatives, UNiGe and UNiGa. We believe that this originates from a delicate competition of ferromagnetic and antiferromagnetic interactions, which is also reflected by the presence of various magnetic phases in the B-T phase diagrams of UNiGa and UNiGe.

In an attempt to discuss the pressure results more quantatively, we have focussed onto the $5 f$-ligand hybridization effects in these compounds. The hybridization causes a delocalization of the $5 f$ electrons, but, since the spin polarization is conserved in the hybridization process, it also causes indirect exchange coupling between $5 f$ moments. Using the tight-binding approximation, it is possible to get a qualitative and sometimes a quantitative estimate of the importance of the $5 f$-ligand hybridization effects in the formation of the uranium magnetic moments. ${ }^{16,17}$ Band-structure calculations within the tight-binding formalism of different compounds have been introduced by Harrison and Froyen for the study of transition-metal compounds. ${ }^{18}$ Later on, they successfully generalized their approach to determine the interaction between $s, p, d$ and $f$ atomic 
orbital. $^{19-21}$ This method in fact a combination of Anderson LMTO theory ${ }^{22}$ and the transition metal pseudo potential theory ${ }^{23}$ and gives as a result the second moment of the f-hybridization band, which can be used as estimate for the delocalization of the U $5 f$ electrons $^{16}$

$$
\left\langle\left(E_{k}-\varepsilon_{f}\right)^{2}\right\rangle=\sum_{i} \frac{n_{i}}{2 l^{\prime}+1} \times\left(V_{l l^{\prime} \sigma}^{2}+2 V_{l l^{\prime} \pi}^{2}+2 V_{l l^{\prime} \delta}^{2}+2 V_{l l^{\prime} \phi}^{2}\right)
$$

where

$$
V_{l l^{\prime} m}=\eta_{l l^{\prime} m} \mathrm{~h}^{2} / 4 \pi^{2} m_{e}\left(r_{l}^{2 l-1} r_{l^{\prime}}^{2 l^{\prime}-1}\right)^{1 / 2} d^{-\left(l+l^{\prime}+1\right)}
$$

are the general hybridization elements between two ions with radii at $r$ and $r^{\prime}$ at distance $d$. The indexes are the angular moments $l, l^{\prime}(l=0,1,2,3$ for $s, p, d$ and $f$ orbital) and the symmetry of the bond $\mathrm{m}(\mathrm{m}=0,1,2,3$ for $\sigma, \pi, \delta, \phi$ bonds $) . m_{e}$ is the mass of the electron, $n_{i}$ is the number of the nearest neighbors with orbital quantum number $l^{\prime}$ and $\eta_{l l^{\prime} m}$ is the general coefficients which are given by ${ }^{24}$

$$
\eta_{l l^{\prime} m}=\frac{(-1)^{l^{\prime}+1}}{6 \pi} \frac{\left(l+l^{\prime}\right) !}{2^{l+l^{\prime}}} \frac{(2 l) !\left(2 l^{\prime}\right) !}{l ! l^{\prime} !}(-1)^{m} *\left[\frac{(2 l+1)\left(2 l^{\prime}+1\right)}{(l+m) !(l-m) !\left(l^{\prime}+m\right) !\left(l^{\prime}-m\right) !}\right]^{1 / 2}
$$

Harrison and $\mathrm{Straub}^{20}$ had derived a criterion for the localization of the magnetic moments due to the Coulomb repulsion U. If the covalent energy (square root of the total hybridization energy) is smaller than $U \sin ^{2}\left(Z_{f} \pi / 14\right)$, with $Z_{f}$ the number of $f$ electrons, localization and thus magnetic order of the $\mathrm{f}$ electrons may be expected. For $\mathrm{U}^{3+}$ (expected for most UTX compounds) the critical energy equals to $1.38 \mathrm{eV}$. We used the above formalism to calculate the different contributions to hybridization energy for the UTX compounds studied here. As can be seen in table 2, the $\mathrm{V}_{\mathrm{ff}}$ contribution is much smaller than the other contributions, which indicate $5 \mathrm{f}-\mathrm{p}$ and $5 \mathrm{f}-\mathrm{d}$ hybridization govern the magnetism in these compounds. For the $\mathrm{ZrNiAl}$ compounds, our data are in reasonable agreement with the results published in ref. 16. Comparing table 2 with table 1 , we find that $\mathrm{p}_{\mathrm{c}}$ and $\mathrm{dT}_{\mathrm{N}} / \mathrm{dp}$ scale reasonably well with the total hybridization, ranging from the local-moment compounds $\mathrm{UNiGa}$ and $\mathrm{UNiGe}$ with lower total hybridization values, passing the more itinerant systems UIrGe, UNiAl toward UCoAl that exhibits the largest degree of itineracy.

In conclusion, we have studied and summarized the effect of pressure on the ordering temperatures and critical fields of various antiferromagnetic UTX compounds ( $\mathrm{T}$ $=\mathrm{Ni}, \mathrm{Co}, \mathrm{Ir} ; \mathrm{X}=\mathrm{Al}, \mathrm{Ga}, \mathrm{Ge}$ ) by means of magnetoresistance studies in magnetic fields up to $18 \mathrm{~T}$. The studies were performed on single crystals under hydrostatic pressure up to $10 \mathrm{kbar}$. By comparing the results with different contributions of the $5 f$-ligand hybridization, which were deduced from tight-binding calculations, we find relatively weak pressure dependence in compounds, where $5 f$ electrons are more localized, while substantially large pressure effects are found in the more itinerant compounds.

\section{Acknowledgments}

The work was supported by a grant from NSF (grant number: DMR-0094241). Work at the National High Magnetic Field Laboratory in Los Alamos was performed under the auspices of the NSF (INT-9722777), the US Department of Energy and the State of Florida. 


\section{References}

${ }^{1}$ V. Sechovsky and L. Havela, in Handbook of Magnetic Materials, edit by K.H.J. Bushchow (North-Holland: Elsevier, Amsterdam, 1998), vol.11, pp 1

${ }^{2}$ H. Nakotte, Ph.D thesis, University of Amsterdam (1994)

${ }^{3}$ K.H.J. Buschow, E. Bruck, R.G. Van Wierst, F. R. de Boer, L Havela, V. Sechovsky, P. Nozar, E. Sugiura, M. Ono, M. Date and A. Yamagishi, J.Apl.Phys. 67, 5212 (1990)

${ }^{4}$ V. Sechovsky, H. Havela, P. Nozar, E. Bruck, F.R. de Boer, A.A. Menovsky, K.H.J. Bushow and and A.V. Andreev, Physica B 136, 103 (1990)

${ }^{5}$ L. Havela, V. Sechovsky, F.R. de Boor, E. Bruck and H.Nakotte, Physica B 177, 159(1992)

${ }^{6}$ V. Sechovesky, L. Havela, F.R. de Boer, P.A. Veenhuizen, K. Sugiyana, T. Kulda, E. Sugiwara, M. Ono, M. Date and A. Yamagishi, Physica B 177, 164 (1992)

${ }^{7}$ N. Kioussis, H.J. Yu, B.R. Cooper, Q.G. Sheng and J.M. Wills, J. Appl. Phys. 73, 5424 (1993)

${ }^{8}$ K.Prokes, T. Fujita, N.V. Mushnikov, S. Hane, T. Tomita, T. Goto, V. Sechovsky A.V. Andreev and A.A. Menovsky, Phys. Rev. B 59, 8720 (1999)

${ }^{9}$ O. Mikulina, J. Kamarad, A. Lacerda, O. Syschenko, T. Fujita, K. Prokes, V. Sechovsky, H. Nakotte, W. Beyerman and A.A. Menovsky, J. Appl. Phys. 87, 5152 (2000)

${ }^{10}$ O. Mikulina, V. Sechovesky, A.H. Lacerda, H. Nakotte, J. Kamarad, W.P. Beyerman, L. Havela and V. Sechovsky, Physica B 281-282, 206 (2000)

${ }^{11}$ F. Honda, T. Kagayama, G.O. Omi, A.V. Andreev, L. Havela, V. Sechovsky, K. Prokes and E. Bruck, J. Phys. Soc. Jpn. 66, 8720 (1997)

${ }^{12}$ A.V. Andreev, M.I. Bartashevich, T. Goto, K. Kamishima, L. Havela and V. Sechovsky, Phys. Rev. B 55, 5847 (1997)

${ }^{13}$ T. Goto, N.V. Mushnikov, H. Yamada, A.V. Andreev andV. Sechovsky, Physica B 281-282, $216(2000)$

${ }^{14}$ K. Prokes, T. Tahara, T. Fujita, H. Goshima, T. Takabatake, M Mihalik, A.A. Menovsky, S. Fukuda and J. Sakurai, Phys.Rev.B, 60, 9532 (1999)

15T. Akazawa, T. Suzuki, T. Tahara, T. Goko, J. Goshima, F. Nakamura, T. Fujita, T. Takabatake and H. Fujii, Physica B 284-285, 261(1999).

${ }^{16}$ K. Prokes, E. Bruck, H. Nakotte, P.F. de Chatel and F.R. de Boer, Physica B 206-207, 8 (1995)

${ }^{17}$ E. Bruck, Ph.D thesis, University of Amsterdam (1991)

${ }^{18}$ W.A. Harrison and S. Froyen, Phys. Rev. B 21, 3214 (1980)

${ }^{19}$ W.A. Harrison, Phys. Rev. B 28, 550(1983)

${ }^{20}$ G.K.Straub and W.A. Harrison, Phys. Rev. B 31, 7668 (1985)

${ }^{21}$ W.A. Harrison and G.K. Straub, Phys. Rev. B 36, 2695 (1987)

${ }^{22}$ O.K. Anderson, Phys. Rev. B 12, 3060 (1975)

${ }^{23}$ W.A.Harrison, Phys. Rev. 181, 1036 (1969)

${ }^{24}$ J.M. Wills and W.A. Harrison, Phys. Rev. B 28, 4363 (1983) 


\section{Figure captions}

Fig. 1: Temperature dependence of the magnetoresistance of UIrGe at $6 \mathrm{kbar}$ for various magnetic fields applied along the orthorhombic $b$ axis.

Fig. 2: Temperature dependence of the electrical resistance of UIrGe at ambient, 6 and 9

kbar pressure. The inset shows the field dependence of the percentage change in the resistivity at 6 and 9 kbar. 


\section{Tables and captions}

Table 1. Ordering temperatures $\left(T_{N}\right)$, pressure rates $\left(d_{N} / d p\right),\left(d B_{c} / d p\right)$ and critical pressure $\left(\mathrm{p}_{\mathrm{c}}\right)$ for various UTX compounds.

\begin{tabular}{|c|c|c|c|c|c|}
\hline Compound & $T_{N}(K)$ & $\begin{array}{c}d T_{N} / d p \\
(K / k b a r)\end{array}$ & $\begin{array}{c}\mathrm{dB}_{\mathrm{c}} / \mathrm{dp} \\
(\mathrm{T} / \mathrm{kbar})\end{array}$ & $p_{c}$ (kbar) & Ref. \\
\hline UIrGe & 14.5 & -0.34 & -.06 & 48 & this \\
\hline UNiGe & $41.5^{*}$ & $<-0.10$ & .24 & $>300$ & 10 and this \\
\hline UNiAl & 17.1 & -0.185 & $-.062^{* *}$ & 105 & $\begin{array}{l}8,9 \text { and } \\
\text { this }\end{array}$ \\
\hline UNiGa & $34.1^{*}$ & -0.11 & .04 & $>300$ & 11 \\
\hline UCoAl & $20^{* * *}$ & - & $.27^{* * *}$ & 24 & 12,13 \\
\hline
\end{tabular}

*other transitions occur at slightly higher temperatures

${ }^{* *}$ derived from extrapolation of pressures below 1 kbar

${ }^{* * *}$ UCoAl forms a metamagnetic ground state

Table 2. Hybridization energy of UTX compounds. $V_{l \prime}$ denote the different types of contributions (see text). In the last column, the sum over all contributions is given.

\begin{tabular}{ccccc}
\hline Compound & $\begin{array}{c}\mathrm{V}_{\mathrm{ff}} \\
(\mathrm{meV})\end{array}$ & $\begin{array}{c}\mathrm{V}_{\mathrm{fd}} \\
(\mathrm{meV})\end{array}$ & $\begin{array}{c}\mathrm{V}_{\mathrm{fp}} \\
(\mathrm{meV})\end{array}$ & $\begin{array}{c}\left(\sum V_{l l^{\prime}}^{2}\right)^{1 / 2} \\
(\mathrm{eV})\end{array}$ \\
\hline UIrGe & 128.4 & 615.8 & 749.1 & 0.978 \\
$\mathrm{UNiGe}$ & 147.1 & 287.4 & 749.3 & 0.816 \\
$\mathrm{UNiAl}$ & 166.0 & 527.1 & 828.0 & 0.995 \\
$\mathrm{UNiGa}$ & 165.8 & 458.7 & 778.1 & 0.918 \\
$\mathrm{UCoAl}$ & 172.7 & 536.43 & 872.1 & 1.04 \\
\hline
\end{tabular}




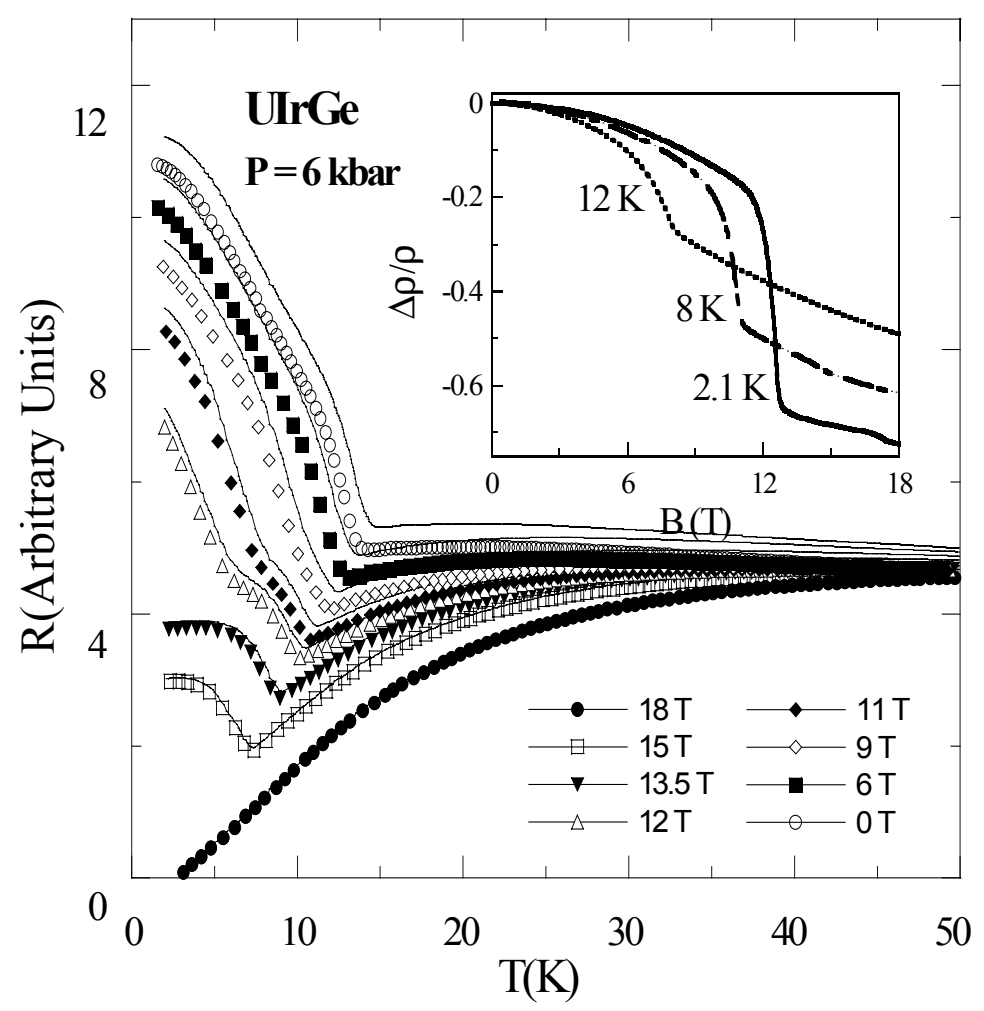




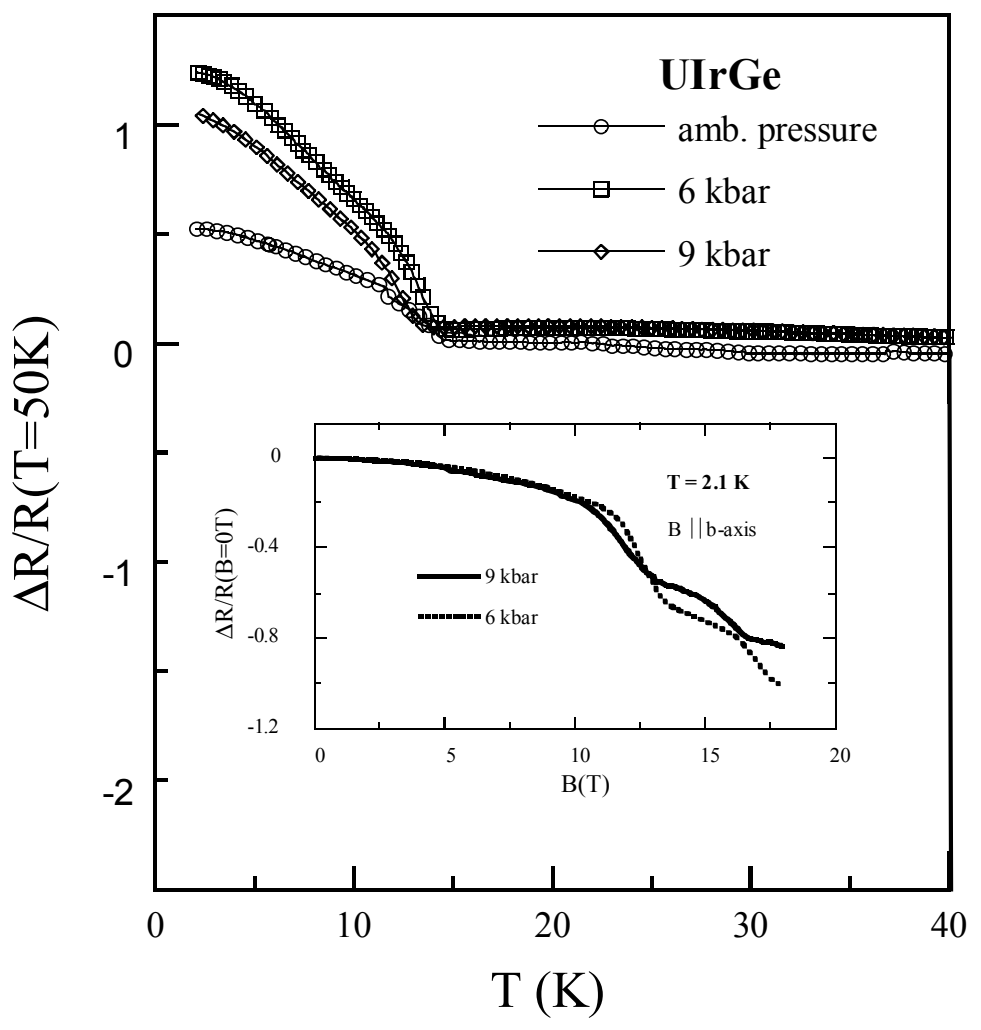

